\title{
Konishi Anomaly and Chiral Gauge Theories
}

\author{
Riccardo Argurio* \\ Physique Théorique et Mathématique \\ Université Libre de Bruxelles, C.P. 231, 1050 Bruxelles, Belgium \\ E-mail: 'Riccardo. Argurio@ulb.ac.bé,
}

Abstract: We begin with a brief introduction on $\mathcal{N}=1$ gauge theories, focusing on the importance of the effective superpotential in light of the new techniques to compute it systematically. We then proceed to consider theories for which the Konishi anomaly proves to be enough to solve exactly for the effective superpotential. As an example we study a chiral $S O(10)$ gauge theory, where we also discuss the occurrence of dynamical supersymmetry breaking.

Gauge theories with minimal $\mathcal{N}=1$ supersymmetry (SUSY) are extensively studied because, while they are believed to display QCD-like properties like confinement, dynamical generation of a mass gap and chiral symmetry breaking, they are still more tractable due to some key SUSY properties like the perturbative non-renormalization theorem and the holomorphy of the effective superpotential. In particular, the SUSY features make it possible to derive some exact results about the vacuum structure of these theories.

The crucial ingredient for deriving such results is, as said above, the holomorphy of the superpotential, both in the fields (possibily effective) and in the couplings [in]. The perturbative non-renormalization theorem then implies that the (Wilsonian) effective superpotential is as follows:

$$
W_{\text {eff }}=W_{\text {tree }}+W_{\text {non-pert }},
$$

that is, only non-perturbative corrections are allowed to take place. We will see that it is precisely these corrections that can be computed in a systematic way. Once that $W_{\text {eff }}$ is known, the exact SUSY vacua can be determined by extremizing it with respect to the low energy effective (gauge invariant) fields that it depends on. (This holds provided there are no singularities in the effective Kähler metric, which are not expected in the cases we will consider below.)

For SUSY gauge theories, one has to assume confinement and generation of a mass gap in the gauge sector. The latter is implemented by the introduction of the (RG invariant)

\footnotetext{
${ }^{*}$ Speaker.
} 
holomorphic scale $\Lambda$, defined in terms of the running gauge coupling $g(\mu)$ and the $\theta$ angle through:

$$
\Lambda^{\beta}=\mu^{\beta} e^{-\frac{8 \pi^{2}}{g^{2}(\mu)}+i \theta},
$$

where $\beta$ is the (positive) coefficient of the one-loop-exact Wilsonian beta function. The matter sector in its turn is described by a set of gauge invariant operators $X_{r}$ which at low energies become the effective fields.

It is then often possible to determine the effective superpotential in the following way. For $W_{\text {tree }}=0$, it can only depend (holomorphically) on the scale $\Lambda$ and on the effective fields $X_{r}$ and it becomes possible to determine the form of $W_{\text {eff }}=W_{\text {non-pert }}\left(X_{r}, \Lambda\right)$ by symmetry arguments alone, and eventually fix the constant factor by an instanton calculation. This approach is based on a case by case analysis of different gauge theories. We will see below that there is a more systematic approach to compute $W_{\text {eff }}$.

We can now add a tree level superpotential, which is typically written in terms of the invariants $X_{r}$ together with their associated couplings, $W_{\text {tree }}=\sum g_{r} X_{r}$. The perturbative non-renormalization theorem and the requirement of good behaviour in several decoupling limits then imply what is often called the "linearity principle" [20, only enter linearly in $W_{\text {eff }}$ :

$$
W_{\text {eff }}=\sum g_{r} X_{r}+W_{\text {non-pert }}\left(X_{r}, \Lambda\right)
$$

namely, no dependence in $g_{r}$ is allowed in $W_{\text {non-pert }}$.

If $W_{\text {tree }}$ gives a mass to some or all of the matter fields, it makes sense to integrate out the massive $X_{r}$. This is done by extremizing ( $(\overline{3}-1)$ with respect to $X_{r}$. The result gives the v.e.v. of $X_{r}$ in terms of all the couplings and $\Lambda$ (assuming that all matter fields have been integrated out). However, by writing the extremization equation as:

$$
g_{r}=-\frac{\partial W_{\text {non-pert }}}{\partial X_{r}}
$$

one sees that the linearity principle implies that integrating out $X_{r}$ is the same as performing a Legendre transform where $X_{r}$ and its coupling $g_{r}$ are a conjugate pair.

One then obtains the effective superpotential in terms of the couplings and the holomorphic scale, $W_{\text {eff }}\left(g_{r}, \Lambda\right)$. Thinking of integrating out as a Legendre transform leads to invert the relation $\left(\begin{array}{l}\mathbf{A}_{1}^{\prime} \\ \mathbf{i}\end{array}\right)$ and integrate in the effective fields through:

$$
X_{r}=\frac{\partial W_{e f f}}{\partial g_{r}} .
$$

The linearity principle guarantees that this procedure is exact as far as the superpotential of the effective theory is concerned. Of course, the relation (歌 for the v.e.v. of a gauge invariant operator also follows from the path integral of the theory.

The ideas above extend to the gauge sector of the theory. Consider the tree level action of the gauge theory, with the coupling running at one-loop. We can write it as the following F-term:

$$
-\beta S \log \frac{\Lambda}{\mu}
$$


where we have introduced the glueball superfield $S$, defined by:

$$
S=-\frac{1}{32 \pi^{2}} \operatorname{tr} \mathcal{W}^{\alpha} \mathcal{W}_{\alpha}, \quad \mathcal{W}_{\alpha}=-\sqrt{2} i \lambda_{\alpha}+\theta^{\beta} F_{\alpha \beta}+\ldots
$$

The term $\left(\overline{\bar{G}_{i}}\right)$ can be interpreted as saying that $S$ and $-\beta \log \Lambda / \mu$ are conjugate in the same way as $X_{r}$ and $g_{r}$. We can then trade the dependence on $\Lambda$ in $W_{\text {eff }}$ into dependence on $S$ by simply integrating in $S$. We compute:

$$
S=\frac{1}{\beta} \Lambda \frac{\partial W_{e f f}}{\partial \Lambda},
$$

and then invert it to reexpress $\Lambda=\Lambda\left(S, g_{r}\right)$.

Finally, we can express the effective superpotential in terms of this new set of variables:

$$
W_{e f f}\left(S, g_{r}, \Lambda\right)=W_{V Y}(S, \Lambda)+W_{p e r t}\left(S, g_{r}\right) .
$$

We have split it into two parts. The first, $W_{V Y}$, is the pure gauge part and takes the

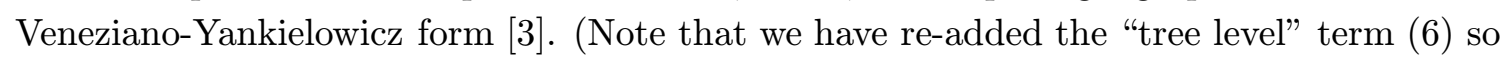
that the holomorphic scale $\Lambda$ appears instead of the cut-off $\mu$.) By extremizing it one gets, for instance for a pure $S U(N)$ theory, the relation $S^{N}=\Lambda^{3 N}$.

The second part, $W_{\text {pert }}\left(S, g_{r}\right)$, can be computed systematically and in a perturbative expansion, as pointed out recently in [4. linearity principle we do not lose any information by integrating out and in again, exactly the same information is contained in $W_{\text {eff }}\left(S, g_{r}\right)$ and in $W_{\text {non-pert }}\left(X_{r}, \Lambda\right)$.

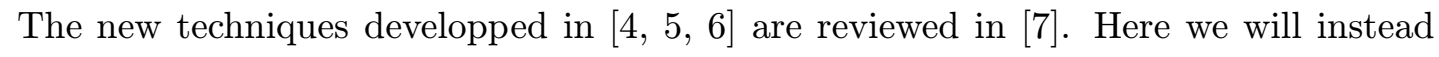
focus on a class of theories for which this approach is at best problematic. Indeed, the approach of [6] relies on the presence of a matter field in the adjoint representation of the gauge group, while the perturbative method of [in], which applies to more generic representations, requires the matter fields to have a mass. Both of these requirements are not met by purely $\mathcal{N}=1$ theories (i.e. with no adjoint) with chiral matter content. ${ }^{1}$

On the other hand, in purely $\mathcal{N}=1$ gauge theories one can see that the Konishi

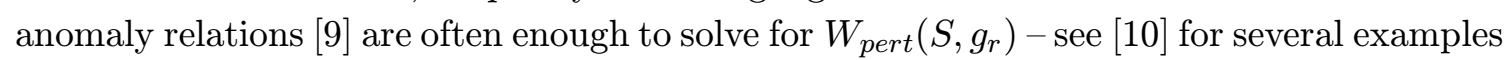
of this, including some particular chiral theories.

Here we will review how the Konishi anomaly can be used to determine the effective superpotential, and then apply the method to a chiral theory which displays dynamical SUSY breaking.

The Konishi anomaly for a $U(1)$ rotation of the superfield $\Phi$ can be roughly written as:

$$
\bar{D}^{2} \Phi^{\dagger} e^{V} \Phi=\sum \# g_{r} X_{r}-\# S,
$$

where on the LHS the action of $V$ is always in the representation to which $\Phi$ belongs, and on the RHS the numerical coefficients depend on the specific theory being considered. The first term is generated by the tree level superpotential, while the second term is generated at one loop. There will be as many relations as there are fundamental matter fields.

\footnotetext{
${ }^{1} \mathrm{~A}$ chiral theory containing also adjoint matter has been considered in $[\overline{8}$. In this case the techniques of [i, $[\overline{6}]$ are applicable.
} 
The highest component of $\left(\overline{1} \overline{1}_{1}^{\prime}\right)$ is nothing else than the usual chiral anomaly. More

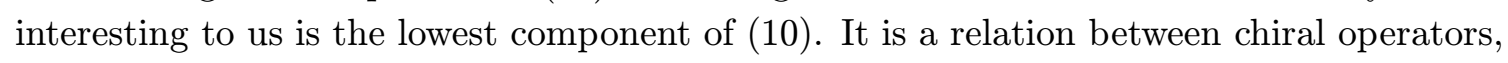
i.e. precisely the class of operators that can have a non-trivial v.e.v. in a SUSY vacuum. However, the chiral operator on the LHS can be written as a SUSY variation of another gauge invariant operator, and thus it trivially has a vanishing v.e.v. in a SUSY vacuum. We are thus left with a set of relations which can be solved for the v.e.v.s of $X_{r}$ in terms of the couplings $g_{r}$ and $S$, the gluino condensate. Once this is done, one can plug these values into the relation $\left(\overline{p_{1}^{\prime}}\right)$ and obtain linear differential equations for the coupling dependent part of $W_{\text {eff }}$, that is $W_{\text {pert }}$. The last step consists in adding the relevant $S$-dependent (VY) superpotential, by considering some limit where the low energy physics simplifies.

It is straightforward to apply this method to analyze, for instance, $S U\left(N_{c}\right)$ SQCD with some flavors, $N_{f}<N_{c}$. By adding a mass term for the quark superfields, one finds an expression for the meson matrix in terms of the mass matrix and $S$, which then, after taking into account the pure $S U\left(N_{c}\right)$ low energy physics, integrating out the glueball superfield and integrating in the meson superfield, leads to the celebrated Affleck-DineSeiberg superpotential:

$$
W_{n o n-p e r t}=\left(N_{c}-N_{f}\right)\left(\frac{\Lambda^{3 N_{c}-N_{f}}}{\operatorname{det} M}\right)^{\frac{1}{N_{c}-N_{f}}} .
$$

Note that this method directly fixes the numerical coefficient, without having to ultimately resort to instanton computations.

We now turn to a different set up, chiral theories which have been argued to display

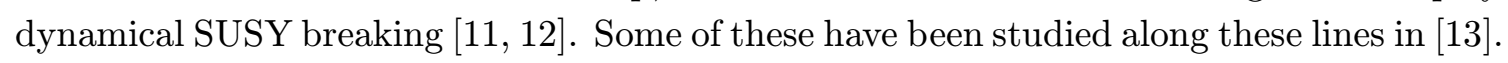
Here we devote our attention to one of the simplest chiral theories, namely $S O(10)$ with one matter field $\Sigma$ in the spinorial 16 representation. This theory is peculiar because there are no invariants that can be written. This means that there is no classical moduli space, but also no way a superpotential, either tree level or non-perturbative, can be written.

One can however write a Konishi anomaly relation as in $\left(\underline{1} \underline{1}_{-1}^{\prime} \overline{0}^{\prime}\right)$ :

$$
\bar{D}^{2} \Sigma^{\dagger} e^{V} \Sigma=-4 S
$$

In a SUSY vacuum, the consequence of (1, $1 \overline{2})$ would be to impose $S=0$. However two lines of arguments can be brought up against this conclusion. Following [1] $\left.{ }_{1}^{1} \overline{1}\right]$, one could argue that in such a symmetric vacuum, anomaly matching of unbroken $U(1)$ global symmetries would imply a highly unlikely SUSY effective theory. Or, in the spirit of [1] ${ }_{-1}^{1} \overline{1}$, a strong coupling instanton computation would yield $\langle S\rangle \neq 0$ in the vacuum.

Both of these lines of arguments lead to removing the assumption that there exists a SUSY vacuum. However since there is no small parameter controlling the SUSY breaking, this is often referred to as dynamical SUSY breaking at strong coupling. Another well known and very similar example is the one of $S U(5)$ with matter in the antisymmetric 10 and anti-fundamental $\overline{\mathbf{5}}$ representations.

At this point we use a trick to make the theory "calculable", also employed in [1] albeit in a different approach. We add a flavor $v$ in the $\mathbf{1 0}$ of $S O(10)$. Now we can write 
two invariants:

$$
X=v^{2} \quad \text { and } \quad Y=v \Sigma^{2},
$$

and we can use them to write a tree-level (renormalizable) superpotential:

$$
W_{\text {tree }}=m X+\lambda Y \text {. }
$$

The Konishi anomaly relations now read:

$$
\begin{aligned}
\bar{D}^{2} v^{\dagger} e^{V} v & =2 m X+\lambda Y-2 S, \\
\bar{D}^{2} \Sigma^{\dagger} e^{V} \Sigma & =2 \lambda Y-4 S .
\end{aligned}
$$

These relations now imply that in a SUSY vacuum $Y=2 S / \lambda$ and, if $m \neq 0, X=0$.

At this point we can either use the equations of motion of the fundamental fields, or use a "classical" generalization of the Konishi relations (where the one-loop term is vanishing) to argue that when $m \neq 0$, we would also have $Y=0$ in a SUSY vacuum, and consequently also $S=0$. To prevent being back in the same situation as before, we take for the time being $m=0$. Thus classically $X$ can take any value and parametrizes the vacuum. On the other hand the Konishi relations fix the v.e.v. of $Y$, so that we can write:

$$
Y=\frac{2 S}{\lambda}=\frac{\partial W_{e f f}}{\partial \lambda}
$$

This is straightforwardly solved as:

$$
W_{\text {eff }}=C(S)+S \log \lambda^{2} .
$$

To determine $C(S)$, we observe that when $X \neq 0$, the gauge symmetry is broken to $S O(9)$. Moreover $\Sigma$ acquires a mass through the tree level superpotential. Thus for large enough v.e.v. $X \gg \Lambda^{2}$, the low energy theory is expected to be pure $S O(9)$ SYM. The full effective superpotential is then:

$$
W_{e f f}=7 S\left(1-\log \frac{S}{\Lambda^{3}}\right)+S \log \lambda^{2} .
$$

It implies for instance that there are 7 SUSY vacua, all of them additionally parametrized by $X$.

We can now integrate in $Y$ and integrate out $S$ to get:

$$
W_{n o n-p e r t}=5\left(\frac{4 \Lambda^{21}}{Y^{2}}\right)^{\frac{1}{5}} .
$$

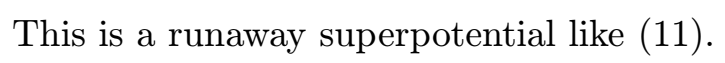

Now we can consider turning on the mass term $m X$ in the tree-level superpotential. Since we know that already classically this term is compatible with a SUSY vacuum only for $Y=0$, that is, precisely at the singularity of (20 $\left(20_{1}^{\prime}\right)$, we see that the extremization of the full superpotential will have no solutions. However, since the mass term lifts all the flat directions, we still expect the potential of the theory to have a non-SUSY minimum, with the location being given in terms of $m$. For small $m$, we expect the minima to be 
located at large values of the invariants $X$ and $Y$, which means that the original $S O(10)$ gauge symmetry is broken at weak coupling (of course here after gauge symmetry breaking a non abelian gauge symmetry subsists, which makes the low energy physics still strongly coupled, unlike the "calculable" theory of [i] $[1] \overline{1}])$. To recover the original model, one takes the limit $m \rightarrow \infty$, so that the matter field $v$ decouples. This simply brings back the SUSY breaking vacuum to a strong $S O(10)$ coupling, thus consolidating (and systematizing) the arguments made before on the theory without the vectorial matter.

To conclude, in this contribution we hope to have convinced the reader that the Konishi anomaly provides a systematic way to derive non-perturbative superpotentials in purely $\mathcal{N}=1$ theories, that is theories where the method [ii] is typically not available.

\section{Acknowledgments}

It is a pleasure to thank Gabriele Ferretti and Rainer Heise for a fruitful collaboration on this and related work, and the organizers of the Johns Hopkins Workshop in Göteborg for giving me the opportunity to present it, and for the nice atmosphere. This work was and is partly supported by EU contracts HPRN-CT-2000-00122 and HPRN-CT-00131, by the "Actions de Recherche Concertées" of the "Direction de la Recherche Scientifique Communauté Française de Belgique", by a "Pôle d'Attraction Interuniversitaire" (Belgium) and by IISN-Belgium (convention 4.4505.86). R.A. is a Postdoctoral Researcher of the Fonds National de la Recherche Scientifique (Belgium).

\section{References}

[1] N. Seiberg, Phys. Lett. B 318 (1993) 469 [arXiv:hep-ph/9309335].

[2] K. A. Intriligator, R. G. Leigh and N. Seiberg, Phys. Rev. D 50 (1994) 1092 [arXiv:hep-th/9403198]; K. A. Intriligator, Phys. Lett. B 336 (1994) 409 [arXiv:hep-th/9407106].

[3] G. Veneziano and S. Yankielowicz, Phys. Lett. B 113 (1982) 231.

[4] R. Dijkgraaf and C. Vafa, arXiv:hep-th/0208048.

[5] R. Dijkgraaf, M. T. Grisaru, C. S. Lam, C. Vafa and D. Zanon, Phys. Lett. B 573 (2003) 138 [arXiv:hep-th/0211017].

[6] F. Cachazo, M. R. Douglas, N. Seiberg and E. Witten, JHEP 0212 (2002) 071 [arXiv:hep-th/0211170].

[7] R. Argurio, G. Ferretti and R. Heise, arXiv:hep-th/0311066.

[8] K. Landsteiner, C. I. Lazaroiu and R. Tatar, arXiv:hep-th/0307182.

[9] K. Konishi, Phys. Lett. B 135 (1984) 439; K. i. Konishi and K. i. Shizuya, Nuovo Cim. A 90 (1985) 111.

[10] A. Brandhuber, H. Ita, H. Nieder, Y. Oz and C. Romelsberger, Adv. Theor. Math. Phys. 7 (2003) 269 [arXiv:hep-th/0303001].

[11] I. Affleck, M. Dine and N. Seiberg, Phys. Lett. B 137 (1984) 187; Phys. Rev. Lett. 52 (1984) 1677; Nucl. Phys. B 256 (1985) 557. 
[12] Y. Meurice and G. Veneziano, Phys. Lett. B 141 (1984) 69; D. Amati, K. Konishi, Y. Meurice, G. C. Rossi and G. Veneziano, Phys. Rept. 162 (1988) 169.

[13] R. Argurio, G. Ferretti and R. Heise, JHEP 0307 (2003) 044 [arXiv:hep-th/0306125].

[14] H. Murayama, Phys. Lett. B 355 (1995) 187 [arXiv:hep-th/9505082]. 